\section{Terapia fotodinámica en el tratamiento de la osteonecrosis inducida por fármacos: reporte de un caso}

\author{
Photodynamic therapy in the treatment of \\ drug-induced osteonecrosis: a case report
}

\section{Resumen}

El objetivo del presente caso fue investigar los beneficios de la terapia fotodinámica (TFD) en ciclos de aplicación como método de tratamiento de la osteonecrosis inducida por fármacos (ONMRM), reportando el caso de un paciente masculino de 75 años, blanco, con diagnóstico de neoplasia maligna renal con metástasis pulmonares y óseas en tratamiento con malato de sunitinib y denosumab. El paciente fue derivado para evaluación dental en un consultorio habilitado para atender pacientes con necesidades especiales, en la ciudad de Salvador-Ba. En el examen intraoral se observaron áreas de tejido óseo expuesto y necrótico en la mandíbula (cara medial) a ambos lados, próximas a la región molar, con aspecto ulcerativo, de tres milímetros de diámetro, y la hipótesis diagnóstica fue de ONMRM en estadio 2. Se inició el protocolo de aplicación de 20 ciclos de TFD, como única forma de tratamiento, en dos sesiones semanales con terapia láser preventiva para la mucositis oral. Durante y al final del tratamiento fue posible el secuestro óseo necrótico, promoviendo la bioestimulación y la curación locorregional, sin manifestación clínica de la patología durante el mantenimiento. Con base en este informe y la evidencia en la literatura científica, se cree que se deben promover los ensayos clínicos, debido a los registros prometedores y efectivos del tratamiento que la terapia hace posible.

Palabras clave: Terapia fotodinámica; Osteonecrosis; Mandíbulas; Metástasis; Denosumab (fuente: DeCS BIREME).

\begin{abstract}
The main of this case was to investigate the benefits of photodynamic therapy (PDT) in application cycles as a method of treatment of medication-related osteonecrosis of the jaw (MRONJ), reporting the case of a 75-year-old white male patient with a diagnosis of renal malignancy with lung and bone metastases treated with sunitinib malate and denosumab. The patient was referred for dental evaluation in an office set up to attend patients with special needs, in the city of Salvador-Ba. In the intraoral examination, areas of exposed and necrotic bone tissue were observed in the mandible (medial aspect) on both sides, close to the molar region, with an ulcerative appearance, three millimeters in diameter, and the diagnostic hypothesis was stage 2 ONMRM The application protocol of 20 PDT cycles was started, as the only form of treatment, in two weekly sessions
\end{abstract}

\section{Caso Clínico}

Rodrigo Araújo Santos ${ }^{1, a, b}$, Norma Lúcia Luz Sampaio 1,a,c, Maria Cecilia Fonseca Azoubel 1,a,d

${ }^{1}$ Escola Bahiana de Medicina e Saúde Pública, Salvador, Bahia, Brasil.

a Cirujano Dentista.

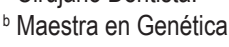

${ }^{c}$ Maestra en Procesos Interactivos de los Órganos y

Sistemas.

${ }^{d}$ Doctora en Ciencias Médicas.

\section{Correspondencia:}

Rodrigo Araújo Santos: rodrigo.uesb@gmail.com Rua Jaldo Reis, número 109, Bairro Centro, Ipiaú, Bahia, Brasil

ORCID: 0000-0002-2921-1747

\section{Coautores:}

Norma Lúcia Luz Sampaio: normaluzs@gmail.com ORCID: 0000-0001-8704-1216

Maria Cecília Fonseca Azoubel: mcfazoubel@gmail.com ORCID: 0000-0003-0325-6370

\section{Editor:}

Juan Carlos Cuevas-González

Universidad Autónoma de Ciudad Juárez, México.

Conflicto de intereses: los autores declaran no tener conflictos de interés.

Fuente de financiamiento: autofinanciado.

Recibido: 26/01/21

Aceptado: 04/06/21

Publicado: 05/10/21 
with preventive laser therapy for oral mucositis. During and at the end of the treatment, necrotic bone sequestration was possible, promoting biostimulation and locoregional healing, without clinical manifestation of the pathology during maintenance. Based on this report and the evidence in the scientific literature, it is believed that clinical trials should be promoted, due to the promising and effective records of the treatment that the therapy makes possible.

Keywords: Photodynamic therapy; Osteonecrosis; Jaws; Metastasis; Denosumab (source: MeSH NLM).

\section{Introducción}

La osteonecrosis de los maxilares es una condición patológica de necrosis ósea de una región debido a la falta y/o interrupción del suministro de sangre, llevándolo a su colapso, así como se le asocia una sintomatología dolorosa y, definido clínicamente por la exposición del hueso necrótico ${ }^{1,2}$. Actualmente, los estudios indican que el uso continuo de algunos medicamentos puede inducir esta condición ${ }^{2,3}$, como los fármacos que actúan sobre la remodelación ósea y la antiangiogénesis ${ }^{2,4}$.

Para clasificar la osteonecrosis inducida por medicamentos (ONMRM) son esenciales tres características: paciente en tratamiento actual o previo con agentes antiangiogénicos o antirreabsortivos; presencia de hueso expuesto, o hueso sondable mediante via fístula, persistente por más de ocho semanas; y, ningún antecedente de radioterapia o metástasis en los maxilares ${ }^{2,3}$. Sin embargo, algunos autores relatan que determinadas manifestaciones clínicas sin exposición ósea como la bolsa profunda, el trismus, el signo de Vincent, entre otras, también se consideran como ONMRM ${ }^{2,4}$.

Los fármacos antirreabsortivos de la clase de los bifosfonatos (en cuatro generaciones) y los inhibidores de RANK-L (denosumab), así como los agentes antiangiogénicos (bevacizumab) se han utilizado en la terapia antitumoral y en diversas enfermedades. No obstante, éstos disminuyen la capacidad fisiológica de la remodelación ósea, lo que conduce a la ONMRM ${ }^{2-7}$.

Se han descrito varias posibilidades para el tratamiento de la ONMRM, y se han propuesto asociaciones de técnicas para estabilizar la evolución de la lesión, entre las que destacan están la terapia antibiótica, la cirugía, la oxigenación hiperbárica, el desbridamiento no quirúrgico, el uso de aceite ozonizado, la pentoxifilina y el tocoferol, el plasma rico en plaquetas, la terapia fotodinámica y la terapia con láser de baja intensidad ${ }^{2,8}$.

La terapia láser, que consiste en el uso de un láser de baja potencia aplicado directamente sobre la lesión; esta es clasificada en láser rojo (660 nanómetros-nm) y láser infrarrojo (780nm). Ambos promueven la estimulación de la síntesis del colágeno, la acción analgésica y antiinflamatoria, diferenciándose en la curación de tejidos blandos y la acción bactericida para el primero y la bioestimulación en la curación de tejidos profundos para el segundo ${ }^{9-11}$.
La terapia fotodinámica (TFD) consiste en el uso de terapia láser de baja potencia en el espectro rojo asociada a un colorante inductor ${ }^{11}$. Esta ha demostrado ser una excelente alternativa para el tratamiento de la ONMRM, debido a sus propiedades biomoduladoras, siendo muy eficiente cuando se asocia a otras modalidades terapéuticas: antibioterapia, debridamiento óseo, cirugía, plasma rico en plaquetas y terapia láser ${ }^{2}$.

Así, ante las prometedoras perspectivas de la TFD, el objetivo de este estudio es presentar un caso de osteonecrosis, manejado con TFD de forma terapéutica combinada con terapia láser para prevenir la mucositis; investigando sus beneficios, así como el protocolo de uso, mostrando los resultados en este caso particular.

\section{Reporte del caso}

Un paciente de 75 años, género masculino, portador de neoplasia maligna de riñón (CIE C64) con metástasis en el pulmón y el hueso, fue indicado por la oncóloga para una evaluación dental. En ese momento estaba tomando Sutent (malato de sunitinib) y Prolia 60mg (denosumab) para prevenir lesiones óseas. El paciente se quejaba de malestar y dificultad para comer y de un intenso dolor en la boca, y que, según la orientación médica del servicio de oncología, estaba tomando acetónido de triamcinolona (oncilon A en orabase); también informó de que el proceso se había iniciado aproximadamente un año antes, cuando había sido sometido a tratamiento oncológico. El examen clínico intraoral reveló áreas de tejido óseo expuesto y necrótico en la mandíbula en fase medial de ambos lados, observandose tres regiones de lesión (figura 1), dos en el lado derecho y una en el izquierdo, correspondientes a la superficie lingual cerca de la región molar derecha e izquierda, respectivamente, con un aspecto inicial ulceroso y que medían alrededor de 3 milímetros de diámetro; la sospecha diagnóstica fue de osteonecrosis inducida por fármacos (ONMRM) en estadio 2, que se caracteriza por la presencia de hueso expuesto y necrótico asociado a infección y dolor según la Asociación Americana de Cirujanos Orales y Maxilofaciales (AAOMS) ${ }^{3}$. Al examen ortopantomográfico fue posible evidenciar áreas radiopacas sin contorno definido en la región afectada (figura 2).

La intervención al paciente se llevó a cabo después de que éste diera su consentimiento mediante un formulario de consentimiento informado (FCI) y manteniendo el contacto con el médico oncólogo, al cual fue solicitada la evaluación sobre la posibilidad de modificar la 

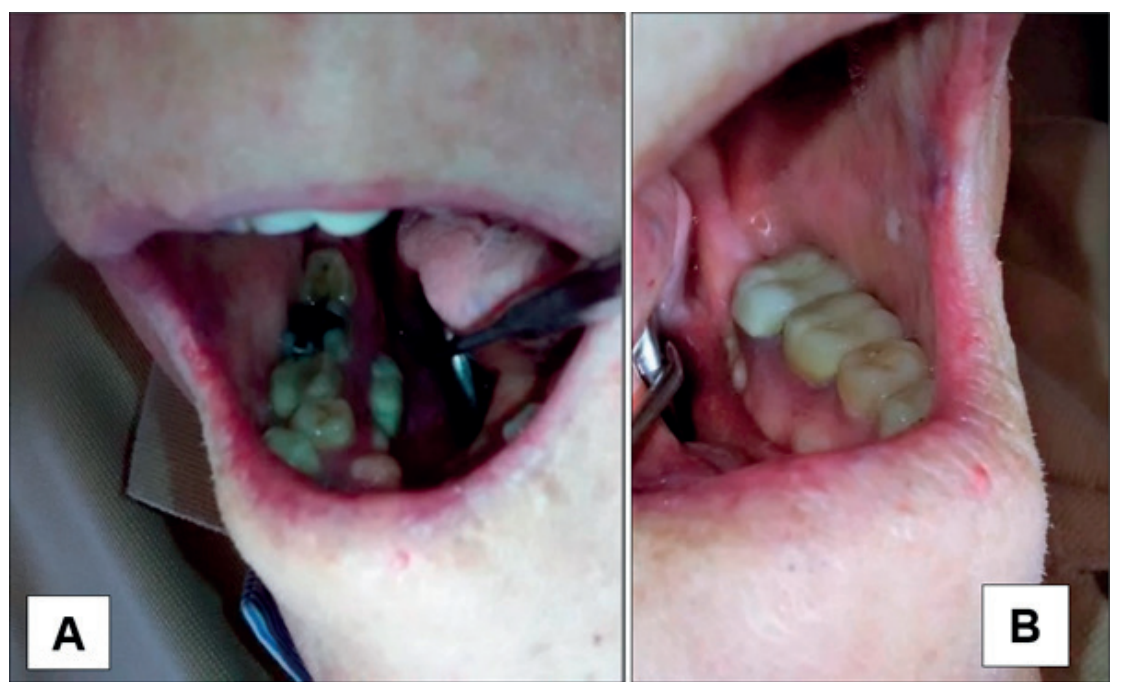

Figura 1. Examen intraoral. A. Región molar derecha. B. Región molar izquierda

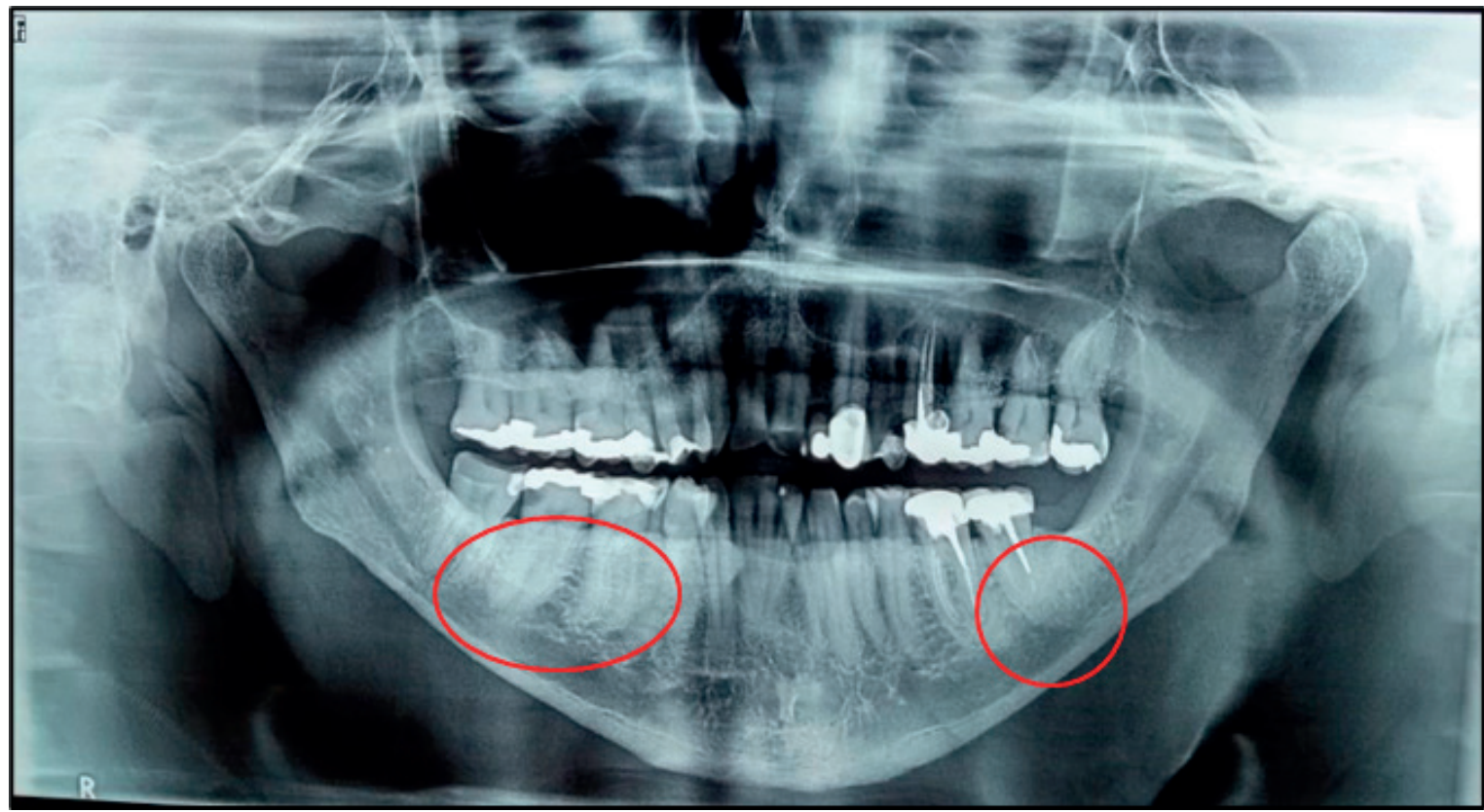

Figura 2. Radiografía panorámica inicial, evidenciando área radiopaca sin contorno definido en la región de las molares de ambos lados

medicación utilizada, a la que se respondió con prontitud. Así, se suspendió la medicación Prolia $60 \mathrm{mg}$ (denosumab) para la mejora del estado general de la paciente. La terapia fotodinámica de las lesiones fue iniciada inmediatamente utilizando azul de metileno al $0,1 \%$ (Chimiolux) con activación por láser de baja potencia (Therapy EC de DMC - Sáo Paulo/SP Brasil, potencia de $100 \mathrm{~mW})$ en la frecuencia roja $(660 \mathrm{~nm})$. Se realizaron 20 ciclos, donde cada ciclo fue conformado por dos sesiones (en dos días -lunes y jueves) con una frecuencia semanal. Se adoptó el siguiente protocolo: 1) aplicación de azul de metileno durante 5 minutos para teñir la región más afectada; 2) irradiación con láser de baja potencia en frecuencia roja $(660 \mathrm{~nm})$; 3) Se establecieron 5 Joules $(\mathrm{J})$ como dosis total en los puntos de aplicación, siendo estos los correspondientes a las zonas del centro y de los bordes de las lesiones; 4) al final, la realización del protocolo preventivo de la mucositis oral con irradiación de láser rojo en toda la boca, con el objetivo de no afectar a la patología, por tratare de un paciente susceptible por su condición sistémica.

Al final del segundo ciclo de TFD ( $3^{\mathrm{a}}$ y $4^{\mathrm{a}}$ sesiones), el paciente informó una notable mejora del dolor, ya siendo capaz de comer adecuadamente. El protocolo se mantuvo, sin embargo, cada vez que se introducía la medicación, el paciente informaba un empeoramiento de los síntomas. Durante el cuarto ciclo ( $7^{a}$ y $8^{a}$ sesiones de DFT) el paciente tuvo que ser hospitalizado con un episodio de trombosis venosa en el miembro inferior y suspendió su tratamiento de TFD durante dos meses. 
La paciente volvió a la consulta, tras 5 meses y el alta médica, con un marcado empeoramiento y al examen clínico intraoral reveló nuevas lesiones en la cara medial de la mandíbula en la región premolar inferior del lado derecho, y el examen complementario fue posible observar un área radiopaca sin contorno definido en la región premolar inferior derecha (figura 3). En la consulta de retorno, la paciente estaba siendo medicada con Pazopanib (clorhidrato de pazopanib) $400 \mathrm{mg}$ (antiangiogénico), Naprix (anlodipino besilato y ramipril) 10 mg (utilizado para el tratamiento del glomérulo renal y la taquicardia) y Marevan (warfarina sódica) $2,5 \mathrm{mg}$ (para la prevención de la trombosis venosa).

El protocolo de terapia fotodinámica se reanudó en julio de 2017. En el sexto ciclo (11 a y $12^{a}$ sesiones de TFD) se produjo la expulsión de secuestros óseos (Figura 4), por biomodulación y regeneración tisular por segunda intención estimulada por la terapia láser, en el lado posterior izquierdo, correspondiente a las lesiones iniciales (mandíbula, región molar del lado izquierdo); en ese momento, los secuestros óseos colectados fueron enviados para un

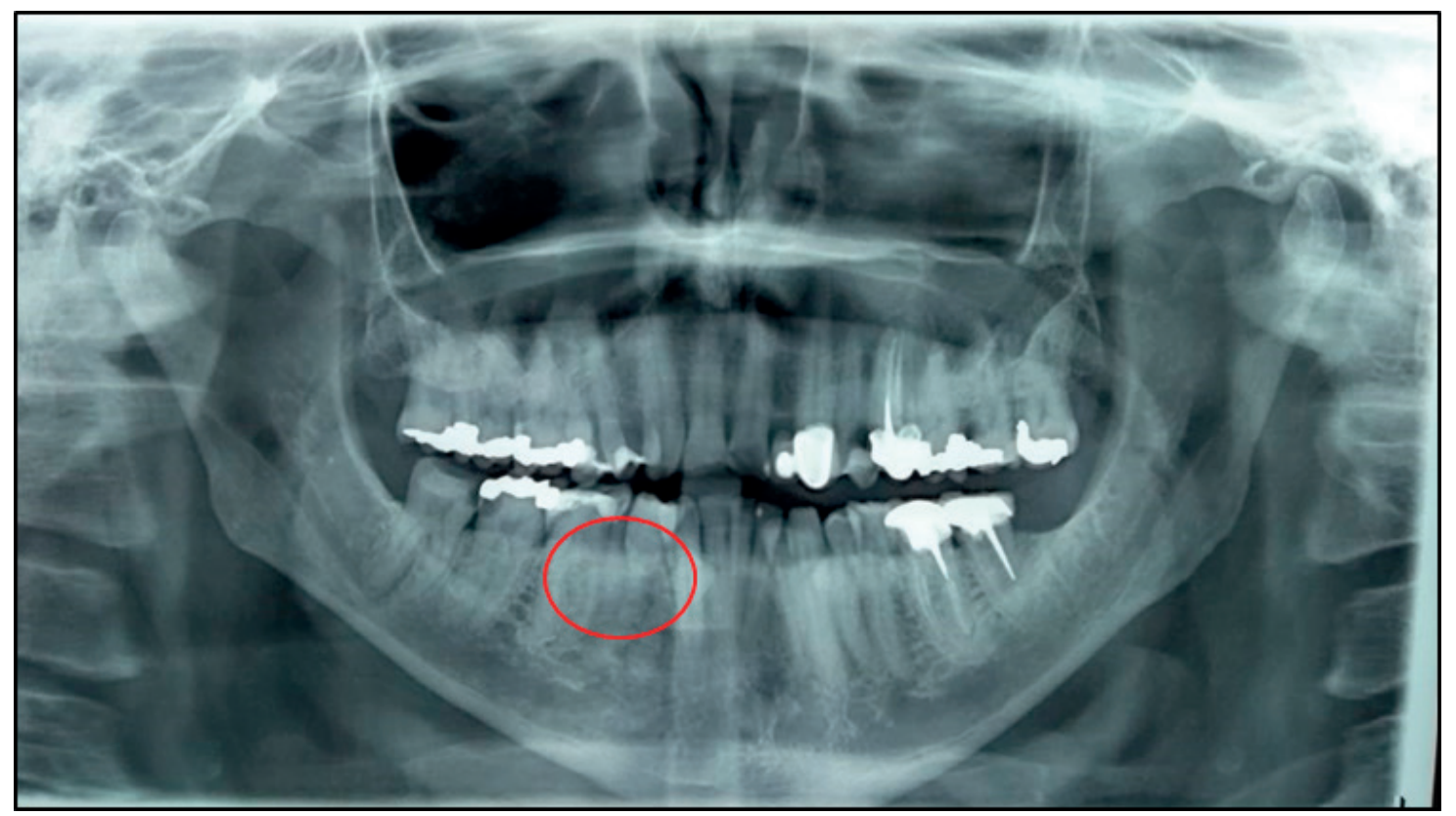

Figura 3. Radiografía panorámica después de 5 meses, evidenciando área radiopaca sin contorno definido en la región de premolares del lado derecho
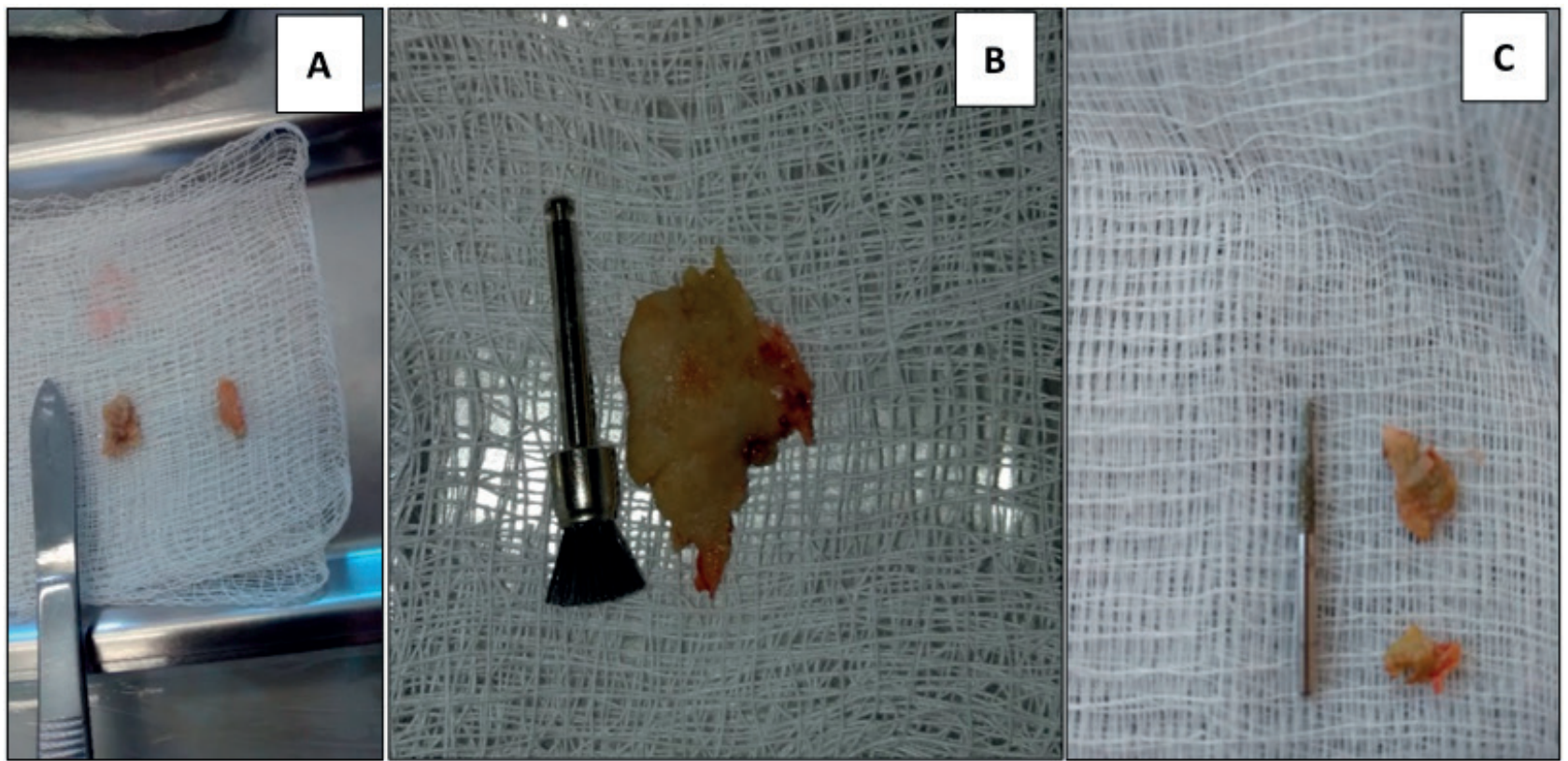

Figura 4. Secuestros óseos colectados. A. Julio de 2017: 6o ciclo - secuestro óseo del lado izquierdo (región MID). B. Septiembre de 2017: 8 ciclo - tercer fragmento óseo, secuestro óseo del lado derecho (región MID). C. Abril de 2018: 11ㅇcclo - cuarto fragmento óseo retirado, secuestro óseo de la región premolar inferior del lado derecho 
examen anatomopatológico. En septiembre de 2017, el tercer fragmento de la región molar del lado derecho, fue removido en el octavo ciclo $\left(15^{\mathrm{a}}\right.$ y $16^{\mathrm{a}}$ sesiones de DFT). En abril de 2018, el cuarto fragmento correspondiente a la región premolar inferior, lesiones tras la consulta de retorno, fue removida en el undécimo ciclo (sesiones 21 y 22 del TFD); concomitantemente, el resultado del examen anatomopatológico confirmó la sospecha diagnóstica inicial de ONMRM.

En las visitas posteriores, se observó una marcada mejoría en el estado de la paciente y, en el examen clínico intraoral, se pudo comprobar la cicatrización de las regiones afectadas correspondientes a las lesiones iniciales y las posteriores a la suspensión del tratamiento (figura 5) con buena respuesta tras la TFD, sin presencia de tejido óseo expuesto o necrótico en toda la región mandibular. La paciente permaneció en mantenimiento y prevención con consultas periódicas de evaluación en consultorio y utilizando clorhidrato de pazopanib (medicamento utilizado para tratar el carcinoma de células renales avanzado y/o metastásico). El paciente fue dado de alta de las citas odontológicas, pues no hubo ninguna manifestación clínica de ONMRM durante un período de un año y tres meses después de la finalización del ciclo de terapia fotodinámica. Debido al avanzado estado de la enfermedad sistémica, el paciente falleció en julio de 2019.

\section{Discusión}

En el caso reportado, los medicamentos utilizados para el tratamiento oncológico de la paciente que indujeron la condición patológica de ONMRM fueron denosumab y clorhidrato de pazopanib. El primero actúa interfiriendo en la unión de las proteínas activadoras de los osteoclastos (Rank-L), no permitiendo la acción osteoclástica, lo que dificulta la regeneración del tejido óseo con el consecuente aumento de su densidad; el segundo actúa sobre el receptor celular del factor de crecimiento vascular, que posee excelentes propiedades con respecto a combatir la proliferación tumoral, pero con repercusiones secundarias sobre la integridad de los microvasos, lo que compromete la nutrición ósea, conduciendo a la necrosis ósea ${ }^{2-7}$.

Los criterios establecidos en la literatura para ser considerados como ONMRM fueron investigados de acuerdo a la Asociación Americana de Cirujanos Orales y Maxilofaciales (AAOMS), esta condición patológica se define por estadios, y estas son guías para ayudar en la elección del tratamiento adecuado, ya que aún no hay instrucciones estandarizadas en la literatura para tal ${ }^{2,3,8}$.

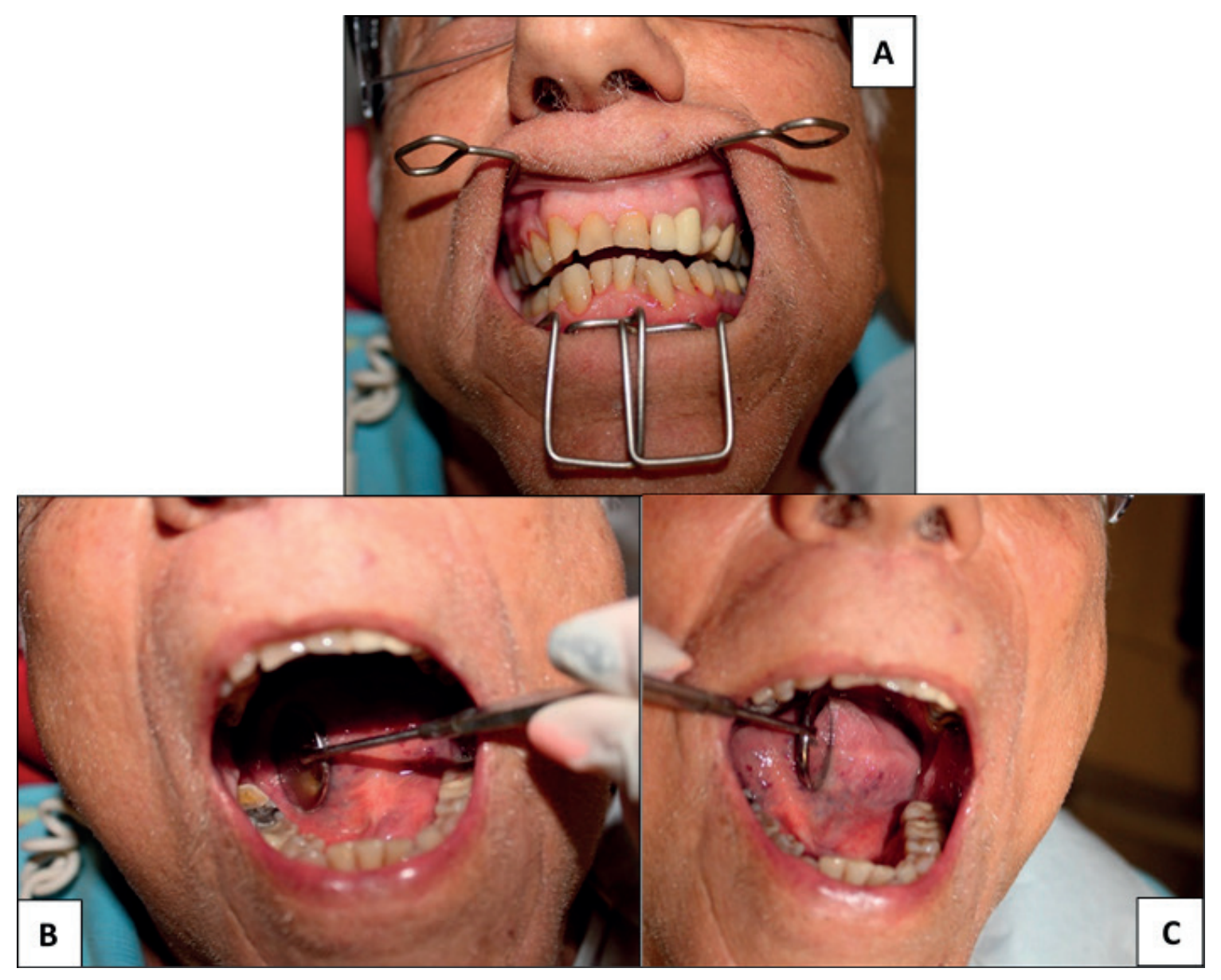

Figura 5. Aspecto intraoral al finalizar el ciclo de terapia fotodinámica. A. Vista exploratoria de la región vestibular. B y C. Cicatrización de las regiones afectadas correspondiente a las lesiones iniciales y posteriores 
En este caso, el paciente fue diagnosticado en el estadio 2 , cuya indicación de tratamiento es únicamente sintomática con antibióticos orales, colutorio bucal antibacteriano, control del dolor y desbridamiento superficial para aliviar la irritación de los tejidos ${ }^{3,12}$. Sin embargo, en el caso presentado, se logró la resolución completa de la infección utilizando únicamente la TFD, así como en el reporte encontrado de un paciente con antecedentes de dosificación mensual de bifosfonatos orales, tomando ácido ibandrónico $150 \mathrm{mg}$ durante 3 años consecutivos para el tratamiento de la osteoporosis, donde sólo esta terapia fotodinámica resultó ser extremadamente eficaz en la resolución de la ONMRM ${ }^{12}$.

El protocolo utilizado en el régimen de tratamiento con TFD para el presente estudio muestra concordancia con otras literaturas, donde los ciclos con aplicación en dos sesiones semanales tienen un potencial de resolución, como en los estudios de Tandon et al. ${ }^{12}$ y Rugani et al. ${ }^{13}$, donde para el primero, la TFD aplicada con un protocolo similar en cinco mujeres en estadio 0 de $\mathrm{ON}$ MRM mostró la cura completa después de las primeras cuatro sesiones y, para el segundo, la TFD con un protocolo similar aplicada en siete pacientes en estadio 2 de ONMRM mostró resolución en cinco pacientes.

Un relato de caso semejante fue descrito por Minamisako et al. ${ }^{14}$ para el tratamiento del estadio 2 de ONMRM, donde su tratamiento consistió en un abordaje conservador con Clindamicina $600 \mathrm{mg} /$ día y la aplicación tópica de gluconato de clorhexidina $(0,12 \%)$, con reuniones quincenales y más sesiones semanales frecuentes, que incluían desbridamiento óseo superficial, aplicación de TFD y terapia con láser (LLLT) por 12 meses, hasta la curación clínica de la exposición ósea, totalizando 37 sesiones. Así, pudieron concluir que la TFD controlaba la infección y la LLLT promovía la reparación total de la mucosa oral, de forma similar al presente caso, en el que, además de la TFD, se utilizó un protocolo preventivo de boca completa para la mucositis oral mediante la aplicación de la terapia láser, teniendo éxito en el tratamiento establecido.

Las ventajas de los tratamientos con láser (rojo e infrarrojo), así como de la TFD, radican en el proceso fotobiológico a traves de la absorción molecular del componente luminoso, incluyendo la descontaminación y la fotoestimulación que acelera la resolución de la enfermedad y la cicatrización de los tejidos blandos, permitiendo, a su vez, la liberación de los factores de crecimiento y la síntesis de colágeno; en la acción vascular, proporciona una mayor oxigenación con formación de nuevos vasos seguido de la vasodilatación local, promoviendo la aceleración del proceso de reparación. Estas características se vuelven esenciales cuando el uso empírico de antibióticos locales y/o sistémicos pueden ser insuficiente para erradicar la formación de biofilm en el hueso expuesto en la ONMRM. Por esta razón, en el caso presentado, la TFD fue utilizada como terapia coadyuvante en la erradicación del biofilm patógeno,ya que los patógenos bacterianos grampositivos y gramnegativos, así como los parásitos, los hongos (con el género
Actinomyces como principal contribuyente) y los virus identificados en la ONMRM son los objetivos de las especies reactivas de oxígeno liberadas durante el proceso fotoquímico de la TFD 9-11,15.

La variación en el régimen de la TFD y el periodo de cicatrización pueden ser atribuidos a la diferencia en el estadio de la lesión de la ONMRM y la diferencia en el tratamiento adyuvante seleccionado. Estudios de Tandon et al. ${ }^{12}$ evidencian que cuando la TFD es aplicada directamente al hueso y en los tejidos infectados, no hay efectos perjudiciales en los tejidos circundantes, corroborando su aplicación en el manejo exitoso de la ONMRM, como se realizó para el presente estudio. Además, informan que aunque la TFD está establecida como un complemento en el tratamiento no quirúrgico, se necesitan pruebas sólidas para dilucidar su papel en el tratamiento y concluyen que la terapia fotodinámica puede ser viable en los estadios avanzados de la ONMRM, que es considerado difícil de tratar y puede ser incluso resistente a la terapia.

El abordaje del tratamiento del ONMRM debe ser realizado por un equipo multidisciplinario, considerando el estado general del paciente y la relación riesgo/beneficio, siendo de suma importancia el conocimiento continuo y actualizado de los profesionales involucrados.

Se debe recomendar siempre las consultas odontológicas periodicas, buscando un buen mantenimiento, es decir, antes, durante y después del tratamiento farmacológico oncológico, debido a los potenciales eventos adversos derivados de los fármacos utilizados en el proceso antitumoral.

Estudios adicionales sobre los beneficios de la TFD como método de tratamiento para este tipo de lesiones óseas son esenciales, así como la optimización de los protocolos utilizados con la finalidad de garantizar un estado no recurrente a la terapia. Para ello, es válido destacar la necesidad de realizar ensayos clínicos robustos, prospectivos y aleatorios acerca de la prometedora terapia empleada en este caso clínico.

\section{Referencias bibliográficas}

1. Mourão CFAB, Moura AP, Manso JEF. Tratamento da osteonecrose dos maxilares associada aos bifosfonatos: revisão da literatura. Rev. Bras. Cir. Cabeça Pescoço. 2013;42(2):113-7.

2. Ribeiro GH, Chrun ES, Dutra KL, Daniel FI, Grando LJ. Osteonecrosis of the jaws: A review and update in etiology and treatment. Braz J Otorhinolaryngol. 2018;84(1):102-8.

3. Ruggiero SL, Dodson TB, Fantasia J, Goodday R, Aghaloo T, Mehrotra B, O'Ryan F. American Association of Oral and Maxillofacial Surgeons position paper on medication-related osteonecrosis of the jaw --- 2014 update. J Oral Maxillofac Surg.2014;72:1938-56.

4. Yoneda T, Hagino H, Sugimoto T, Ohta H, Takahashi S, Soen S, Taguchi A, Nagata T, Urade M, Shibahara T, Toyosawa S. Antiresorptive agent-related osteonecrosis of the jaws: Position Paper 2017 of the Japanese Allied 
Committee on Osteonecrosis of the Jaw. J Bone Miner Metab. 2017;35(1):1-14.

5. Clézardin P. Mechanisms of action of bisphosphonates in oncology: a scientific concept evolving from antiresorptive to anticancer activities. Bonekey Rep. 2013; 2(January):1-7.

6. Santos-Silva AR, Belizário Rosa GA, Castro Júnior G De, Dias RB, Prado Ribeiro AC, Brandão TB. Osteonecrosis of the mandible associated with bevacizumab therapy. Oral Surg Oral Med Oral Pathol Oral Radiol. 2013;115(6):e32-6.

7. Sivolella S, Lumachi F, Stellini E, Favero L. Denosumab and anti-angiogenetic drug-related osteonecrosis of the jaw: An uncommon but potentially severe disease. Anticancer Res. 2013;33(5):1793-8.

8. Magremanne M, Reychler H. Pentoxifylline and tocopherol in the treatment of yearly zoledronic acid-related osteonecrosis of the jaw in a corticosteroid-induced osteoporosis. J Oral Maxillofac Surg. 2014;72(2):334-7.

9. Mesquita KS, Queiroz AM, Nelson-Filho P, Borsatto MC. Terapia fotodinâmica: tratamento promissor na odontologia ? Fac Odontol lins/Unimep. 2013;23(2):45-52.

10. Minamisako MC, Grando LJ, Meurer MI, Guterres Y, Girardi, C. Abordagem de osteorradionecrose mandibular com laserterapia de baixa potência. Rev Gaúcha Odontol. 2014; 62(Suppl. 0):113.
11. Kwiatkowski S, Knap B, Przystupski D, Saczko J, Kędzierska E, Knap-Czop K, et al. Photodynamic therapy - mechanisms, photosensitizers and combinations. Biomedicine \& Pharmacotherapy. 2018;106:1098-1107.

12. Tandon S, Lamba AK, Faraz F, Aggarwal K, Chowdhri $\mathrm{K}$. A case report of bisphosphonate related osteonecrosis of the jaw treated by photodynamic therapy. Photodiagnosis Photodyn Ther. 2019;26(April):313-5.

13. Rugani P, Truschnegg A, Acham S, Kirnbauer B, Jakse N. Use of Photodynamic Therapy in Treatment of Bisphosphonate-related Osteonecrosis of the Jaws: Literature Review and Case Series. J Anal Bioanal Tech. 2013;S1:006.

14. Minamisako MC, Ribeiro GH, Lisboa ML, Mariela Rodríguez Cordeiro M, Grando LJ. Medication-Related Osteonecrosis of Jaws: A Low-Level Laser Therapy and Antimicrobial Photodynamic Therapy Case Approach. Case Rep Dent. 2016; 2016. DOI: 10.1155/2016/6267406

15. Poli PP, Souza FÁ, Maiorana C. Adjunctive use of antimicrobial photodynamic therapy in the treatment of medication-related osteonecrosis of the jaws: A case report. Photodiagnosis Photodyn Ther. 2018;23:99-101. 